\title{
La formación de paraísos de contaminación: un estudio de caso de la producción de celulosa en el Cono Sur
}

Fernanda Queiroz-Sperotto. Fundação de Economia e Estatística, Porto Alegre, Brasil.

RESUMEN | En las últimas tres décadas han ocurrido importantes cambios de localización de segmentos industriales altamente contaminantes que migran de países desarrollados a países en desarrollo. Una de las hipótesis investigadas es la formación de paraísos de contaminación. Para contribuir con la investigación de esos paraísos, el artículo propone un análisis acerca de la conducta y del desempeño ambiental de empresas extranjeras de celulosa, localizadas en la región del Cono Sur. La investigación identificó ligeras diferencias de conducta, pero significativas de desempeño. En general, las plantas extranjeras en el Cono Sur presentan un mejor desempeño. Sin embargo, los datos de exportación indicaron una especialización en los intercambios comerciales. Por lo tanto, es posible afirmar que hay en la región un proceso inminente de paraísos de contaminación, en razón de la instalación de tantas plantas industriales de gran tamaño, muchas con capacidad productiva superior a un millón de toneladas/año.

PALABRAS CLAVE | gestión ambiental, indicadores ambientales, localización.

ABSTRACT | In the last three decades, important changes to the location of highly polluting industrial segments have occurred (from developed countries to developing countries). One of the investigated hypotheses of this paper is the formation of pollution havens. To contribute to the research of these havens, the article proposes an analysis of behavioral and environmental performance of foreign companies cellulose, located in the Southern Cone Region (Argentina, Brazil, Chile and Uruguay). The research identified subtle differences in behavior, but significant in performance. In general, foreign plants in the Southern Cone have a better performance. Moreover, export data indicated a specialization in trade. Therefore, it can be said that in the region there is an imminent pollution havens process, due to the installation of many industrial plants of large size, many with more than one million tons/year production capacity.

KEYWORDS | environmental management, environmental indicators, location. 


\section{Introducción}

El ritmo más acelerado de producción y consumo vivido en las últimas tres décadas hizo que la problemática ambiental ganase mayor peso en el debate internacional. En ese contexto, uno de los fenómenos investigados fue la deslocalización de sectores de elevado potencial de contaminación de países desarrollados a países en desarrollo. La idea central es que las empresas estarían optando por una estrategia de localización motivada por la perspectiva de costos de control ambiental menores, asociados a reglas ambientales más permisivas. Esa es la base de la hipótesis de paraísos de contaminación (HPC). Para contribuir en el debate acerca de la formación de esos paraísos, la presente investigación analiza si el intenso crecimiento del sector de celulosa en el Cono Sur -Argentina, Brasil, Chile y Uruguay- no estaría siendo motivado por reglamentos ambientales poco exigentes y, así, contribuyendo a la formación de un paraíso de contaminación. Para responder a esa cuestión, el análisis privilegió informaciones de conducta y desempeño ambiental de las compañías de celulosa -extranjeras y nacionales- localizadas en la región del Cono Sur.

A continuación, en la segunda sección, se discuten algunos aspectos acerca de la temática ambiental y de las estrategias de localización industrial. En especial, se presentan algunas dificultades relacionadas a la observación de la HPC y, a partir de esas, se propone un modelo de evaluación de indicadores ambientales (o evaluación ambiental). En la sección tres, se exponen brevemente las características del sector de celulosa, con énfasis en las estadísticas de producción, exportación e importación. En la cuarta, se aplica la metodología de evaluación de desempeño y de conducta ambiental en dos grupos de plantas industriales: uno formado por las empresas extranjeras ubicadas en la región y sus filiales en el extranjero; y otro por las primeras y las empresas nacionales. Finalmente, en la quinta, se presentan las consideraciones finales.

\section{Medioambiente y localización industrial}

En los ańos 1970 hubo importantes iniciativas y resoluciones acerca del real efecto de las actividades productivas sobre el medioambiente. En esa década fue publicado el informe Limites del Crecimiento, elaborado por el Club de Roma, y hubo la primera gran conferencia internacional sobre el medioambiente - la Conferencia de Estocolmo- que resultó en la creación del Programa de las Naciones Unidas para el Medio Ambiente (pnuma). Además de sensibilizar a los países acerca de la necesidad de adoptar criterios y principios comunes de defensa del medioambiente, se trataron cuestiones de gran relevancia, como los impactos del subdesarrollo y el retraso tecnológico entre los países desarrollados y los en desarrollo.

La mayor importancia de las cuestiones ambientales resultó en la elaboración y adopción de reglamentos más estrictos y, por consiguiente, en el crecimiento de los gastos en controles de contaminación, los cuales pasaron a formar parte de los costos de producción, de localización, de adquisición de bienes de capital y de administración. En ese sentido, el reglamento ambiental se ha convertido en una condición de localización, porque, de acuerdo con la jurisdicción política, cambia 
según contenido y compromiso, la distribución geográfica de las industrias contaminantes tiende a ser desigual y los costos para aplicar las reglas cambian de un lugar a otro y de una actividad a otra (Hayter, 1997; Stafford, 1985). Por lo tanto, el cumplimiento de las reglas de protección ambiental pasa a afectar la distribución espacial de la industria, alterando, por ejemplo, las tendencias de aglomeración y desaglomeración, los potenciales de desarrollo industrial de las regiones, los procesos de decisión de localización por parte de las empresas y las percepciones públicas y privadas sobre el espacio (Stafford, 1977).

Sin embargo, los resultados de algunas investigaciones acerca de los efectos del reglamento ambiental en la localización industrial -en su mayoría elaborados a partir de cuestionarios y modelos econométricos- señalaron pocas evidencias de esa interferencia (Birdsall \& Wheeler, 1993; Brunnermeier \& Levinson, 2004; Chapman, 1982; Dean, 1992; Dean, Lovely \& Wang, 2009; Gray, 2002; Jänicke, Binder \& Mönch, 1997; Leonard, 1988; Levinson, 1996; Low \& Yeats, 1992; Lyne, 1990; Mulatu, Gerlagh, Rigbi \& Wossink, 2010; ocDE, 2002; Porter, 1999; Smarzynska \& Wei, 2001; Zarsky, 1999).

Una parte de esas investigaciones trata del problema a partir de la hipótesis de la formación de paraísos de contaminación (HPC). El enfoque se centra en la relación entre los cambios de comercio e inversiones y los reglamentos ambientales, dando énfasis a la deslocalización de sectores industriales con elevado nivel de contaminación. Esa hipótesis ganó peso a finales de los años 1970, cuando se intensificaron los flujos de inversión extranjera directa (IED) en los países en desarrollo. La preocupación era que las industrias con elevado potencial contaminante podrían estar migrando a países con reglamento ambiental supuestamente menos exigente, con la finalidad de mantener su rentabilidad y ventaja competitiva y, así, intentar minimizar los altos costos derivados del cumplimiento de normas ambientales más estrictas.

Aunque la HPC sea razonable desde el punto de vista económico, porque abre una posibilidad a la reducción de los costos con control de contaminación, su evidencia aún no ha sido ampliamente comprobada. Hay por lo menos siete aspectos que podrían verse como obstáculos para comprobar la existencia de paraísos de contaminación. El primero reside en la elección del método de investigación. La mayoría de los investigadores optan por aplicar cuestionarios y entrevista, los cuales están sujetos a parcialidad en su enfoque o desarrollo, ${ }^{1}$ o utilizan datos secundarios (algunos aplicando incluso modelos econométricos) de diversas actividades contaminantes, que muchas veces tienen características de producción muy distintas (Chapman, 1982; Dean et al., 2009; Jänicke et al., 1997; Levinson, 1996; Meyer, 1995; Zarsky, 1999). Segundo, muchos de esos análisis no consideran el efecto de las interferencias de grupos de defensa ambiental y de la relación entre gobierno y empresa. En particular, es conveniente analizar el perfil de funcionamiento legal de las instituciones (públicas o privadas) para identificar, por ejemplo, si existe corrupción. La ausencia de informaciones consistentes, principalmente las que se refieren

1 La aplicación de cuestionarios puede no ser la más adecuada, porque se investiga, indirectamente, la conducta de la empresa con relación al medioambiente, lo que, por razones éticas, siempre debe ser preservado. Además, la retórica corporativa suele estar presente en encuestas como esas (Chapman, 1982). 
a las prácticas de negociación, como la adopción de incentivos a las IED, también puede dificultar la constatación de la HPC (Gray, 2002; Smarzynska \& Wei, 2001). Tercero, existen dificultades para evaluar y mensurar la fuerza de las leyes de protección ambiental entre los distintos países o regiones, así como para comprobar su real aplicación (Smarzynska \& Wei, 2001; Zarsky, 1999). Cuarto, aunque los costos de control ambiental no sean despreciables, con gran frecuencia otros factores de localización son considerados más relevantes, como salarios, niveles de sindicalización, oferta de infraestructura, acceso a los mercados de insumos y de bienes finales, etcétera (Gallagher, 2008). Quinto, es poco probable que los países amenacen a sus industrias con reglas ambientales más severas, que estimularían la deslocalización. Igualmente, en coyunturas económicas menos favorables, la demanda de más protección se reduce y las políticas pasan a atender las necesidades del mercado, incluso las de las industrias más contaminantes (Jänicke et al., 1997). Sexto, en los países en desarrollo es necesario tener en cuenta que la trayectoria de su crecimiento industrial es influenciada por una división internacional de trabajo, que les impone una mayor participación en la producción de bienes más tradicionales (menos tecnológicos) e intensivos en recursos naturales (Birdsall \& Wheeler, 1993; Gray, 2002). Siete, es posible que la formulación de la pregunta esté equivocada, o sea, en lugar de buscar una relación positiva entre los flujos de IED y la adopción de políticas ambientales menos exigentes, la indagación alternativa sería observar si los países, con la intención de atraer las IED, no estarían flexibilizando sus leyes ambientales, utilizándolas como moneda de $\operatorname{cambio}^{2}$ (Gray, 2002). Así, la presencia de estos aspectos, además de hacer más difícil las investigaciones, podría reforzar la tendencia a rechazar la HPC (Sperotto, 2014a; Smarzynska \& Wei, 2001).

A pesar de las dificultades para la verificación de la HPC, muchos esfuerzos analíticos continúan aplicándose en esa dirección, porque no se puede negar que, en menor o mayor grado, hay regiones más sensibles a la concentración de actividades industriales de elevado potencial contaminante. A fin de contribuir a esa discusión, a continuación se propone otro enfoque basado en dos criterios que buscan evitar algunos de los obstáculos para comprobar la HPC.

\section{Evaluación sector-control-conducta-desempeño}

A partir de las dificultades observadas en varias investigaciones que trataron los efectos de la adopción de reglas ambientales más rigurosas en la estrategia de localización de las industrias, en particular las que aplicaron la HPC, se propone un enfoque fundamentado en dos criterios.

El primero centra el análisis en un sector de alto potencial de contaminación: celulosa de mercado. Así, además de evitar problemas de comparación de actividades de producción muy distintas, el sector elegido -como se planteará en la próxima sección -pasó en las últimas dos décadas por cambios significativos en su geografía

2 Tal proposición de flexibilización de las reglas ambientales se conoce en la literatura como race to the bottom o carrera hacia el fondo. La expresión consiste en el ablandamiento de las normas y leyes ambientales por parte de los gobiernos con la finalidad de atraer más recursos de IED, manteniendo o elevando, en su área de jurisdicción, la participación de determinadas industrias, las cuales se ven directamente afectadas por esos reglamentos (Gallagher, 2008; Gray, 2002; Zarsky, 1999). 
de producción. Su producción, que era muy concentrada en economías desarrolladas, en los últimos veinte años se expandió rápidamente en algunas economías en desarrollo, incluso con la presencia de capital extranjero.

El segundo criterio busca una alternativa para el problema de comparación de reglas y normas de protección ambiental entre países o regiones. Como ya se mencionó, de un modo general, la verificación del grado de rigidez de las leyes depende no solo de sus directrices, sino también de su aplicabilidad. La alternativa propuesta es utilizar las prácticas ambientales de las empresas como proxies de medida de rigidez ambiental. El propósito es verificar si hay diferencias de comportamiento ambiental entre las unidades de producción extranjeras -ubicadas dentro o fuera de la región de estudio- y las nacionales, diferencias que podrían constituir supuestos de la HPC. Además, se considera que el uso de esas informaciones públicas valoriza el principio de la transparencia y el uso positivo de instrumentos de comunicación de conducta ambiental.

Para la construcción del modelo se emplearon tres tipos de indicadores ambientales: (i) de control, en el cual se determinan previamente los niveles de emisión; (ii) de conducta, que capta las prácticas y las acciones de las empresas; y (iii) de desempeńo, que indica los niveles efectivos de emisiones de las unidades de producción.

El indicador de control está representado por la evaluación Best Available Technique (вАT). La aplicación de los parámetros de emisión BAT en los controles de proceso es bastante difundida y se fundamenta en tres nociones: (i) best/mejor, que significa las formas o medios más eficientes para alcanzar un elevado nivel de protección ambiental como un todo; (ii) available technique/técnica disponible, que indica las técnicas desarrolladas a una escala que posibilite su aplicación en una clase económica, en condiciones técnicamente viables, considerando los costos y los beneficios, si son, o no, técnicas usadas y producidas dentro del país y si son razonablemente accesibles para el técnico (persona o equipo) que realiza la actividad; y (iii) techniques/técnicas, que incluye la tecnología utilizada y la forma en que las instalaciones son proyectadas, construidas, conservadas, exploradas y encerradas. Esos parámetros son utilizados en la Comunidad Europea para fijar límites de emisión (máximos y mínimos) e imputar penalidades y multas ${ }^{3}$ (European Commission, 2013).

Los otros dos indicadores - conducta y desempeño- siguen la distinción sugerida por Abreu (2001) en su modelo de Estructura-conducta-desempeño-ambiental. Según la investigadora, los indicadores de conducta ambiental buscan demostrar la cualidad de la gestión ambiental de las empresas, en diversas actividades y funciones de gerencia. La selección de esos indicadores sigue la orientación de la Organización

3 Aunque los parámetros definidos por la BAT sean computados para el contexto europeo, se considera que su aplicación en la región del Cono Sur es posible, porque en su cálculo se admiten aspectos diversos, como el tamaño de la planta, las características geográficas y el tipo de madera utilizada. Por ejemplo, en esa evaluación se consideran plantas industriales ubicadas tanto en los países escandinavos como en los países ibéricos, ambas sujetas a condiciones geográficas distintas y, en ese caso, también consumidoras de tipos de madera variados: mientras en Finlandia y Suecia prevalece la producción de celulosa de fibra larga, en España y Portugal se usa exclusivamente el eucalipto, un árbol de fibra corta. De este modo, en la ausencia de parámetros específicos de la región, los límites sugeridos por la BAT son adecuados para cotejar las diferencias de las unidades y de las empresas, extranjeras y nacionales, localizadas en el Cono Sur o en otras regiones. 
para la Cooperación y el Desarrollo Económicos ( $\mathrm{OCDE}$ ), en la cual se encuentran: la norma de la Organización Internacional de Normalización, Iso-14001, que trata de la reglamentación del sistema de gestión ambiental y el indicador de dispendios en inversiones ambientales. También se agregaron a ese grupo la norma Iso 9001 de gestión de calidad y las certificaciones de manejo y de cadena de custodia aplicadas en la exploración de productos forestales: Forest Stewardship Council (FSC) y Program for Endorsement of Forestry Certification (PEFC). En ese último se incluyen la certificación brasileña de productos forestales (Cerflor) y la certificación chilena de productos forestales (Certfor), ambas habilitadas por el PEFC.

Los indicadores de desempeńo corresponden al lado mensurable de la adopción de prácticas y acciones de equilibrio ambiental a partir de los reales niveles de emisión. Siguiendo también la dirección de la ocDE, Abreu (2001) presenta un conjunto de varios índices según cinco dimensiones: aire, agua, suelo, recursos naturales y fauna-flora. ${ }^{4}$ Entre ellos fueron seleccionadas las emisiones en el aire y en el agua observadas en la producción de pulpa y aplicados en el indicador de control BAT-200 I. La tabla 1 presenta el resumen de los indicadores de conducta y de desempeño empleados en esa investigación.

TABLA I | Síntesis de las variables analizadas, según grupo de informaciones

\begin{tabular}{|c|c|c|c|}
\hline & INFORMACIONES & VARI & ABLES \\
\hline \multirow{6}{*}{$\begin{array}{l}\text { Desempeño } \\
\text { ambiental }\end{array}$} & \multirow{2}{*}{$\begin{array}{l}\text { Emisiones en el } \\
\text { aire (BAT) }\end{array}$} & Dust (kg/Adt) - Partículas sólidas & $\begin{array}{l}\text { NOx }(\mathrm{kg} / \mathrm{Adt}) \text { - Dióxido de } \\
\text { Nitrógeno }\end{array}$ \\
\hline & & SO2 (kg/Adt) - Dióxido de azufre & $\begin{array}{l}\text { TRs }(\mathrm{kg} / \mathrm{Adt}) \text { - Compuesto } \\
\text { reducido de azufre }\end{array}$ \\
\hline & \multirow{4}{*}{$\begin{array}{l}\text { Emisiones en el } \\
\text { agua (BAT) }\end{array}$} & Flujo efluentes (m3/Adt) & $\begin{array}{l}\text { TSS }(\mathrm{kg} / \mathrm{Adt}) \text { - sólidos } \\
\text { suspendidos totales }\end{array}$ \\
\hline & & $\begin{array}{l}\text { DQO (kg/Adt) - Demanda quími- } \\
\text { ca de oxígeno }\end{array}$ & P total $(\mathrm{kg} / \mathrm{Adt})$ - Fósforo total \\
\hline & & $\begin{array}{l}\text { BOD }(\mathrm{kg} / \mathrm{Adt}) \text { - Demanda bioquí- } \\
\text { mica de oxígeno }\end{array}$ & $\mathrm{N}$ total $(\mathrm{kg} / \mathrm{Adt})-$ Nitrógeno total \\
\hline & & $\begin{array}{l}\text { AOx }(\mathrm{kg} / \mathrm{Adt}) \text { - halógeno orgánico } \\
\text { absorbido }\end{array}$ & \\
\hline \multirow{5}{*}{$\begin{array}{l}\text { Conducta } \\
\text { ambiental }\end{array}$} & \multirow{3}{*}{ Certificación } & ISO 9OO I & Forest Stewardship Council - FSC \\
\hline & & ISO I 400 I & $\begin{array}{l}\text { Program for Endorsement of } \\
\text { Forestry Certification - PEFC }\end{array}$ \\
\hline & & OHSAS I 8OO I & \\
\hline & \multirow{2}{*}{$\begin{array}{l}\text { Informe de } \\
\text { Sustentabilidad } \\
\text { (Is) }\end{array}$} & \multirow{2}{*}{$\begin{array}{l}\text { Divulgación Informes de Susten- } \\
\text { tabilidad }\end{array}$} & $\begin{array}{l}\text { Datos de emisiones de } \\
\text { contaminación }\end{array}$ \\
\hline & & & $\begin{array}{l}\text { Información sobre inversiones en } \\
\text { controles de contaminación }\end{array}$ \\
\hline $\begin{array}{l}\text { NOTA } \\
\text { FUENTE }\end{array}$ & NA & $\begin{array}{l}\text { UE SIGNIFICA } \\
\text { ГO (2OI } 4)\end{array}$ & LOSA SECA EN EL AIRE. \\
\hline
\end{tabular}


Con el objetivo de contextualizar los cambios de la geografía de producción de celulosa en los últimos veinte años, la próxima sección propone un análisis más particular en la región del Cono Sur —-Argentina, Brasil, Chile y Uruguay-, destacando su reciente expansión y características de esa actividad productiva.

\section{El segmento de celulosa de mercado}

La celulosa es un material fibroso hecho por medios químicos o mecánicos, o también por la combinación de ambos. Está presente en la madera y en otras materias primas celulósicas. En el mercado hay dos tipos de pulpa según el tamańo de la fibra: la corta, proveniente de árboles de madera más dura, y la larga, oriunda de árboles coníferos. El tipo de la fibra y la especie del árbol afectan directamente la calidad y el precio de la pulpa. Las fibras largas permiten una celulosa de mejor calidad y más resistente a las transformaciones fabriles de sus derivados; por lo tanto, son las más valoradas. En la cima de la clasificación de calidad está la celulosa de fibra larga elaborada por los países escandinavos y Canadá. Los precios fijados por esos países son referencia internacional para el sector. En un segundo nivel está la pulpa de fibra larga obtenida de árboles como el pino -Chile es uno de los más importantes productores-. En el tercer grado se encuentra la celulosa de pulpa corta de eucalipto, producida en Brasil. Hay aún un cuarto grupo formado por celulosa mixta, hecha con maderas de varios tipos, incluyendo, también, otras materias primas celulósicas. Normalmente, esas son negociadas a precios menores que las demás (Sperotto, 2016).

Aunque haya tres procesos de producción -químico, mecánico y térmico-, las grandes plantas industriales de pulpa utilizan el químico. Mundialmente, el sector industrial de celulosa presenta una estructura oligopólica. La formación de ese oligopolio ocurre por la presencia de barreras de entrada como: (i) la elevada inversión inicial con maquinarias y equipos; (ii) las economías de escala, por la necesidad de obtener una escala mínima de eficiencia productiva; (iii) la disponibilidad de un macizo forestal; y (iv) la creación de viveros y laboratorios propios para el desarrollo de plántulas para obtener árboles de gran productividad (Biazus, Da Hora \& Leite, 2010; Sperotto, 2012). Especialmente las elevadas inversiones en maquinarias y equipos, junto con la necesidad de formación de grandes macizos forestales, son aspectos que dificultan la entrada de nuevos productores. Además, por ser una commodity de bajo valor agregado y dirigida al mercado mundial, su viabilidad económica está asociada directamente a la escala producción. Justamente, una de las principales ventajas del Cono Sur es la elevada productividad de sus forestas (tabla 2). El aprovechamiento del eucalipto y del pino en la región -rotación y rendimiento- es mucho mayor que en otras regiones.

La demanda de pulpa se vincula predominantemente al consumo de papel y, así, responde directamente a los cambios en el crecimiento económico. Su oferta se ve determinada por el tipo de fibra, la capacidad de producción, el volumen de stocks, la interferencia de costos de transporte y las variaciones de las tasas de cambio, entre otras variables. Según los datos de la Organización de las Naciones Unidas para la Alimentación y la Agricultura (FAO), en 2015 se produjeron 181 millones de toneladas de celulosa. Los mayores productores son Estados Unidos, Canadá, China, Brasil, Suecia y Finlandia, responsables del 70\% de la producción de pulpa. 
TABLA 2 Rotación y rendimiento de los árboles, según tipo de fibra, en países seleccionados

\begin{tabular}{|c|c|c|c|c|}
\hline & PAÍSES & ESPECIES & $\begin{array}{c}\text { ROTACIÓN } \\
\text { AÑos }\end{array}$ & $\begin{array}{c}\text { RENDIMIENTO } \\
\text { M3/HA/AÑo }\end{array}$ \\
\hline \multirow{9}{*}{ Fibra corta } & Brasil & Eucalipto & 7 & 44 \\
\hline & Uruguay & Eucalipto & 08 -oct & 25 \\
\hline & Sudáfrica & Eucalipto & 08 -oct & 20 \\
\hline & Chile & Eucalipto & 10-dic & 25 \\
\hline & Argentina & Eucalipto & 10-dic & 25 \\
\hline & Portugal & Eucalipto & dic-15 & 12 \\
\hline & España & Eucalipto & dic-15 & 10 \\
\hline & Suecia & Betulla & $35-40$ & 6 \\
\hline & Finlandia & Betulla & $35-40$ & 4 \\
\hline \multirow{9}{*}{ Fibra larga } & Brasil & Pino $s s p$ & 15 & 38 \\
\hline & Chile & Pino radiata & 25 & 22 \\
\hline & Nueva Zelanda & Pino radiata & 25 & 22 \\
\hline & Estados Unidos & Pino elliottiiltadea & 25 & 10 \\
\hline & Canadá (costa) & Pino de Oregon & 45 & 7 \\
\hline & Canadá (interior) & Picea glauca & 55 & 3 \\
\hline & Canadá (este) & Picea mariana & 90 & 2 \\
\hline & Suecia & Picea abies & $70-80$ & 4 \\
\hline & Finlandia & Picea abies & $70-80$ & 4 \\
\hline
\end{tabular}

FUENTE ASSOCIAÇÃo BRASIlEira de CELUlose E PAPEL [BRACELPA] (2OI 3 )

En una breve perspectiva, se observan, en los últimos cuarenta años, importantes cambios en la geografía de la celulosa. Aunque en las primeras posiciones estén Estados Unidos y Canadá, después de los años 2000 surgieron nuevos actores e importantes inversiones de grandes compañías europeas (suecas y finlandesas), especialmente en las economías en desarrollo. Es el caso de países como Brasil, Chile, Indonesia e India. En 1970, Brasil era el $13^{\text {er }}$ productor mundial, y en 2015 pasó a ser el 20; Chile, que era el $24^{\circ}$ en 1970, en 2014 llegó al 9o; Indonesia e India, en 1970 , ocupaban la $50^{\text {er }}$ y la $34^{\text {a }}$ posiciones, pero en 2015 subieron a la $8^{\mathrm{a}}$ y a la $10^{\mathrm{a}}$, respectivamente. Los países escandinavos, líderes en la producción de pulpa, permanecieron en los lugares $4^{\circ}$ y $5^{\circ}$.

El mayor volumen importado de celulosa se destina a las economías desarrolladas, entre las cuales se destacan, en Europa, Alemania, Italia, Francia y Holanda; en América del Norte, Estados Unidos; y en Asia, Japón. En el grupo de los países emergentes están China, la mayor importadora mundial, y Corea del Sur. Entre 2010 y 2015, las importaciones chinas de celulosa correspondieron al 30\% de las importaciones totales. En 2015, el volumen total importado fue de 52,9 millones de toneladas, evaluadas en us $\$ 34,4$ mil millones. 
En las exportaciones, la oferta mundial de pulpa de madera es segmentada según los tipos de fibras y tiene como principales proveedores, en orden decreciente de producción: (i) fibra larga: Estados Unidos, Canadá, Chile, Alemania y Noruega; (ii) fibra corta: Brasil, Indonesia, Estados Unidos, Chile, Canadá, Espańa y Portugal. Considerando la producción total de celulosa, Brasil es, desde 2013, el primer exportador mundial en volumen (10,6 millones de toneladas en 2015) y el segundo en valor (us $\$ 4,9$ mil millones). En especial, la región del Cono Sur -Argentina, Brasil, Chile y Uruguay- exportó, en 2015, 16,9 millones de toneladas, correspondientes al 31,5\% del volumen total exportado. Una información que confirma la importante posición del Cono Sur en el mercado mundial de celulosa es el resultado de la balanza comercial; esto es, la diferencia entre exportaciones e importaciones. Según las estadísticas de la FAO, el Cono Sur es la región con mayor superávit de volumen en los cambios comerciales de pulpa (figura 1). En 2015, el resultado fue de 16,3 millones de toneladas. Entre los cuatro países se destaca Brasil, que presentó un saldo de comercio de 10,2 millones de toneladas. Chile es el segundo, con un superávit de 4,7 millones de toneladas. Uruguay es el tercero, con una balanza de comercio de celulosa de 1,5 millones de toneladas.

\section{FIGURA I | Balanza de comercio de celulosa según regiones seleccionadas,} 1980-2015

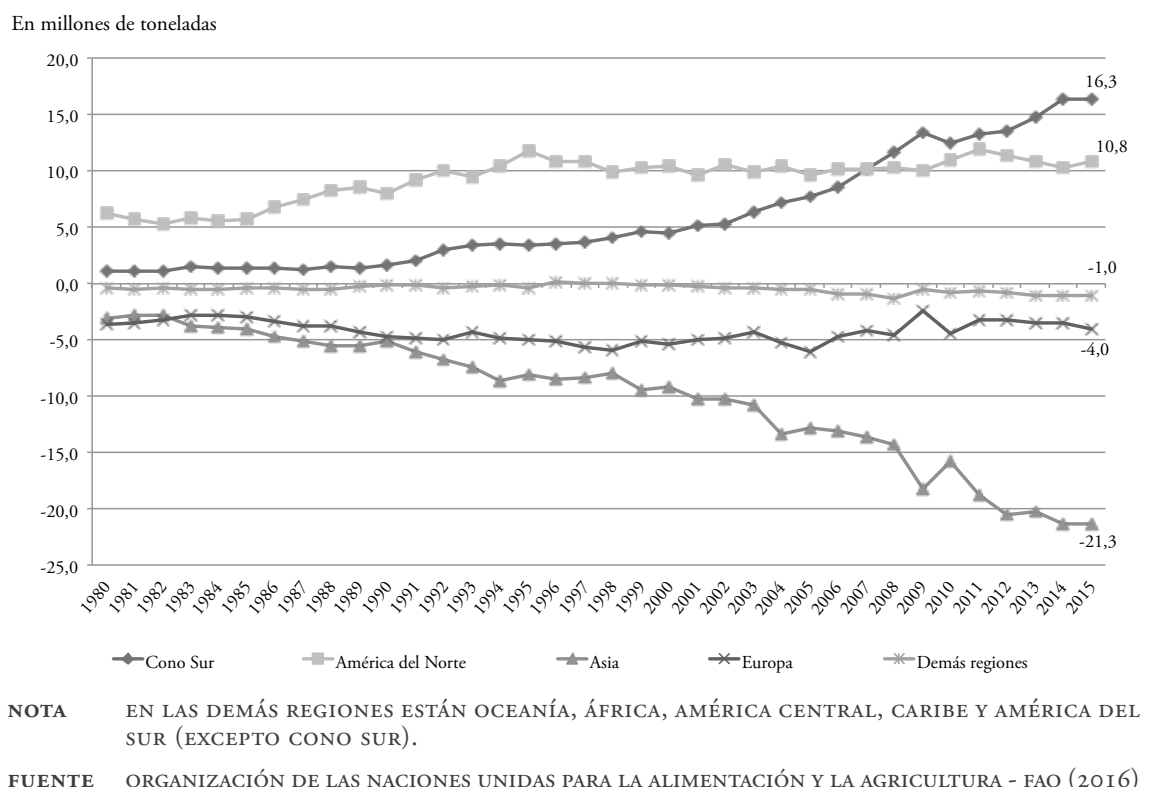

La expectativa para los próximos años es que la posición del Cono Sur en el mercado mundial se consolidará aún más, pues se prevén nuevas inversiones que elevarán la capacidad instalada nominal de la región. Hasta 2020 deberá ocurrir un crecimiento del $34 \%$ de la capacidad instalada brasileńa, que sobrepasará los actuales 15,8 millones de toneladas/ańo y llegará a las 21,2 millones de toneladas/año 
(Sperotto, 2014a; 2014b; 2016). Un dato que llama la atención es el perfil de esas inversiones. Los proyectos actuales, tanto las nuevas plantas industriales como de ampliación de aquellas en operación, son de unidades de producción de capacidad instalada nominal de más de un millón de toneladas/año. La presión por una elevada escala de producción reconfiguró el tamaño de las plantas industriales y pasó a exigir un volumen mayor de madera para abastecer las fábricas. ${ }^{5}$ En este punto hay una gran preocupación respecto de los efectos ambientales de esos complejos industriales.

\section{Aplicación del modelo: el caso de la celulosa en el Cono Sur}

Esta sección tiene como objetivo analizar las diferencias de conducta (prácticas y acciones) y desempeño (niveles de emisiones) ambiental entre las empresas de celulosa del Cono Sur, incluyendo, en el caso de las subsidiarias extranjeras, la comparación con las demás plantas industriales ubicadas en otros países. Como se presentó en la tabla 2, los datos fueron agrupados en dos conjuntos -desempeño ambiental y conducta ambiental-y las informaciones se refieren al año 2012.

El análisis de las diferencias de conducta y de desempeño ambiental a partir de las variables seleccionadas, se aplicó a dos grupos de empresas: uno formado por las empresas extranjeras ubicadas en la región y sus filiales en el extranjero; y otro por las primeras y las empresas localizadas en el Cono Sur. La finalidad es observar si existen diferencias de conducta y de desempeño entre las unidades que puedan indicar la formación de paraísos de contaminación; vale decir, si los niveles de emisión en las unidades ubicadas en países desarrollados son más tolerables que aquellos verificados en las unidades ubicadas en la región del Cono Sur, en la cual se presupone la existencia de reglas ambientales más flexibles. Para el propósito de comparación, el grupo de unidades de producción extranjeras está compuesto exclusivamente por las tres únicas compañías que operaban, en 2013, en la región: Japan Brazil Paper and Pulp Resources Development Co. Ltd. (Jвp Resources Development Co. Ltd), Stora Enso Oyj y upm-Kymmene Oyj. ${ }^{6}$

En la primera parte de esa sección se analizarán los parámetros de desempeño y conducta ambiental de las unidades industriales de las empresas extranjeras. Posteriormente, se focalizarán esos mismos parámetros, pero solamente de las plantas productoras ubicadas en el Cono Sur.

\section{Perfil de las empresas de celulosa extranjeras y sus subsidiarias del Cono Sur}

En 2013, tres empresas extranjeras actuaban en el Cono Sur a través de tres subsidiarias: Cenibra, Veracel Celulose (joint venture con Fibria Celulose s.A.) y UPM Fray Bentos (tabla 3). Además de esas tres plantas, también se seleccionaron todas las otras unidades industriales pertenecientes a las compañías citadas, pero ubicadas

5 En Brasil, una unidad de producción con capacidad instalada de 1,5 millón de toneladas/año de celulosa necesita de 140 mil hectáreas de forestas, siendo que un hectárea tiene, en media, 1.500 árboles de eucalipto (Indústria Brasileira de Árvores [ıв́́], 2015).

6 En 2014 empezó a operar la fábrica Montes del Plata, una joint venture de las compañías Arauco y Stora Enso. 
en otros países; ${ }^{7}$ ellas son Kaukas, Kymi y Pietarsaari, pertenecientes a la compañía upM-Kymmene Oyj; y Enocell, Sunilla y Skutskär, de la compañía Stora Enso Oyj. ${ }^{8}$

El principal tipo de celulosa de esas plantas es la blanqueada kraft de fibra corta. Ese es también el principal tipo fabricado en el Cono Sur: las tres plantas de empresas extranjeras ubicadas en la región producen celulosa de fibra corta. ${ }^{9}$ De las seis plantas ubicadas en Finlandia y Suecia, cuatro ya producen celulosa de fibra corta y larga. Aunque las base de datos consultadas no especifican el volumen según el tipo de celulosa, es muy probable que las plantas extranjeras en el Cono Sur se estén beneficiando con los cambios de conocimiento técnico y, por lo tanto, apropiándose del know-how desarrollado en la producción de celulosa a partir de árboles de madera más dura, como el eucalipto.

TABLa 3 Compañías extranjeras con filiales en el Cono Sur, según unidad, localización y capacidad instalada, 2013

\begin{tabular}{|l|l|l|c|}
\hline \multicolumn{1}{|c|}{ COMPAÑIAS } & \multicolumn{1}{|c|}{ UNIDADES } & \multicolumn{1}{c|}{ LOCALIZACIÓN } & $\begin{array}{c}\text { CAPACIDAD } \\
\text { INSTALADA (T) }\end{array}$ \\
\hline $\begin{array}{l}\text { JBP Resource and } \\
\text { Development Co. }\end{array}$ & Cenibra & Brasil & 1.190 .000 \\
\hline \multirow{4}{*}{ Stora Enso Oyj } & Veracel & Brasil & 1.200 .000 \\
\cline { 2 - 4 } & Enocell & Finlândia & 450.000 \\
\cline { 2 - 4 } & Sunilla & Finlândia & 375.000 \\
\cline { 2 - 4 } & Skutskär & Suécia & 550.000 \\
\hline \multirow{3}{*}{ UPM-Kymmene Oyj } & Fray Bentos & Uruguai & 1.100 .000 \\
\cline { 2 - 5 } & Kaukas & Finlândia & 740.000 \\
\cline { 2 - 4 } & Kymi & Finlândia & 570.000 \\
\cline { 2 - 4 } & Pietarsaari & Finlândia & 790.000 \\
\hline
\end{tabular}

FUENTE INFORMES DE SUSTENTABILIDAD Y PÁGINAS INSTITUCIONALES EN INTERNET

El primer parámetro de evaluación es el índice de partículas sólidas en el aire, Dust (tabla 4). Entre las plantas industriales, solamente las ubicadas en el Cono Sur comunicaron sus índices. Además de esas, se cuenta con la información consolidada de la UPM-Kymmene. ${ }^{10}$ En el Cono Sur, llaman la atención los niveles

7 La excepción es la JPв Resources Development Co. Ltd., con la cual no fue posible establecer una comparación directa por no tener otras unidades de producción de celulosa.

8 En ese grupo de plantas industriales la mayoría produce exclusivamente celulosa. Solamente las unidades Kaukas y Kymi producen celulosa y papel; sin embargo, una parte de la pulpa se destina a la venta.

9 Hasta los ańos noventa, las empresas ubicadas en el hemisferio norte producían en mayor escala la celulosa de fibra larga. Sin embargo, en los últimos años, la producción de pasta de fibra corta se expandió en Europa, influenciada principalmente por la producción portuguesa y española. Actualmente, una parte de la celulosa de los países escandinavos ya es de fibra corta.

10 En el informe de 2012, la upM-Kymmene publica el valor de esas emisiones en toneladas. El dato por toneladas de celulosa no fue calculado porque la empresa no informó la producción individual de las unidades. 
emitidos por la unidad Cenibra, muy elevados. Las otras dos plantas extranjeras en la región emitieron partículas al aire dentro del límite вAт. Las emisiones de dióxido de azufre $\left(\mathrm{SO}_{2}\right)$ y de compuesto reducido de azufre (TRs) son las que provocan el olor desagradable comúnmente asociado a la producción de celulosa. En el caso del $\mathrm{SO}_{2}$ se observa que dos de las unidades extranjeras en el Cono Sur -Veracel y UPM-Fray Bentos- emitieron cantidades inferiores a las fijadas por la BAT. Ya la unidad de Cenibra lanzó un valor seis veces mayor que el definido por la BAT. Otras con niveles superiores fueron Skutslär y Sunilla de la Stora Enso, y Kaukas, de la UPM-Kymmene. Las emisiones de TRs, tanto en el exterior como en el Cono Sur, fueron inferiores al intervalo mínimo definido por la вAт, indicando un desempeño superior al tolerable. Las emisiones de dióxido de nitrógeno $\left(\mathrm{NO}_{\mathrm{x}}\right)$, asociadas a la producción industrial y la quema general de combustible, sobrepasaron el límite tolerable en la mayoría de las plantas. Solamente las fábricas de Cenibra y Veracel, en el Cono Sur, presentaron índices menores que los determinados.

TABla 4 | Índices de emisión en el aire, unidades de producción seleccionadas, 2012

\begin{tabular}{|l|c|c|c|c|}
\hline \multicolumn{1}{|c|}{$\begin{array}{c}\text { PLANTAS INDUSTRIALES/ } \\
\text { COMPAŃíns }\end{array}$} & $\begin{array}{c}\text { DUST (KG/ } \\
\text { ADT) }\end{array}$ & $\begin{array}{c}\text { SO2 (KG/ } \\
\text { ADT) }\end{array}$ & $\begin{array}{c}\text { NOX (KG/ } \\
\text { ADT) }\end{array}$ & TRS (KG/ADT) \\
\hline Cenibra / JBP Resources Develp. Co & 1,31 & 2,61 & 0,133 & 0,02 \\
\hline $\begin{array}{l}\text { Veracel / joint venture Stora Enso y } \\
\text { Fibria }\end{array}$ & 0,23 & 0,009 & 0,806 & 0,006 \\
\hline Fray Bentos / UPM-Kymmene Oyj & 0,13 & 0,05 & 1,6 & 0,004 \\
\hline Enocell / Stora Enso Oyj & NI & 0,35 & 1,92 & NI \\
\hline Sunila / Stora Enso Oyj & NI & 0,49 & 1,74 & NI \\
\hline Skutskär / Stora Enso Oyj & NI & 1,69 & 1,86 & NI \\
\hline Kaukas / UPM-Kymmene Oyj & NI & 0,78 & 1,5 & 0,025 \\
\hline Kymi / UPM-Kymmene Oyj & NI & 0,04 & 1,7 & 0,03 \\
\hline Pietarsaari / UPM-Kymmene Oyj & NI & 0,22 & 1,3 & 0,06 \\
\hline Stora Enso (datos consolidados) & NI & 0,822 & 1,788 & NI \\
\hline UPM (datos consolidados) & 0,1 & 0,3 & 2 & 0,03 \\
\hline Media plantas en el Cono Sur & 0,557 & 0,89 & 0,846 & 0,01 \\
\hline Media plantas en el exterior & 0,1 & 0,586 & 1,726 & 0,036 \\
\hline BAT - límite inferior & 0,2 & 0,2 & 1 & 0,1 \\
\hline BAT - límite superior & 0,5 & 0,4 & 1,5 & 0,2 \\
\hline
\end{tabular}

NOTA ADT ES LA MEDida DE TONELADAS DE CELUlOSA SECA EN EL AIRE. Ni: DATO NO INFORMADO. FUENTE INFORMES DE SUSTENTABILIDAD Y PÁGINAS INSTITUCIONALES EN INTERNET

Para las emisiones en el agua, la evaluación BAT computa siete indicadores. Uno de ellos es el que mide los flujos de agua (tabla 5). En ese ítem, el desempeño de las plantas extranjeras en el Cono Sur fue superior a los observados en las unidades en el exterior. En las tres fábricas del Cono Sur, los valores obtenidos estuvieron por 
debajo del límite superior. En el parámetro de demanda química de oxígeno (DQO) ${ }^{11}$ se observa que todas las plantas depositaron residuos industriales en cantidades inferiores al intervalo superior definido por la BAT. Además, el desempeño de las plantas del Cono Sur, en comparación con las unidades en el exterior, fue mejor. La información de demanda bioquímica de oxígeno (Dво ${ }^{12}$ fue divulgada solamente por las unidades de producción del Cono Sur y sus niveles de emisión estuvieron dentro de los límites tolerables.

TABLA 5 | Índices de emisión en el agua, unidades de producción seleccionadas, 2012

\begin{tabular}{|c|c|c|c|c|c|c|c|}
\hline $\begin{array}{c}\text { PLANTAS } \\
\text { INDUSTRIALES/ } \\
\text { COMPAŃÍAS }\end{array}$ & $\begin{array}{c}\text { FLUJO } \\
\text { EFLUENTE } \\
\text { (M3/ADT) }\end{array}$ & $\begin{array}{l}\text { DQO } \\
\text { (KG/ } \\
\text { ADT })\end{array}$ & $\begin{array}{c}\text { DBO } \\
\text { (KG/ADT })\end{array}$ & $\begin{array}{c}\text { AOX } \\
\text { (KG/ADT) }\end{array}$ & $\begin{array}{c}\text { TSS } \\
\text { (KG/ADT) }\end{array}$ & $\begin{array}{c}\text { P (KG/ } \\
\text { ADT) }\end{array}$ & $\begin{array}{c}\text { N (KG/ } \\
\text { ADT) }\end{array}$ \\
\hline $\begin{array}{l}\text { Cenibra / JPB Re- } \\
\text { sources Develp. Co }\end{array}$ & 42,4 & 8,8 & 0,99 & 0,07 & 1,9 & 0,004 & 0,001 \\
\hline $\begin{array}{l}\text { Veracel / joint venture } \\
\text { Stora Enso y Fibria }\end{array}$ & 21,2 & 5,5 & 0,23 & 0,05 & 0,56 & 0,017 & 0,052 \\
\hline $\begin{array}{l}\text { Fray Bentos / UPM- } \\
\text { Kymmene Oyj }\end{array}$ & 19,3 & 4 & 0,14 & 0,03 & 0,2 & 0,02 & 0,05 \\
\hline $\begin{array}{l}\text { Enocell / Stora } \\
\text { Enso Oyj }\end{array}$ & 55,5 & 19,4 & NI & 0,15 & NI & 0,01 & 0,11 \\
\hline $\begin{array}{l}\text { Sunila / Stora Enso } \\
\text { Oyj }\end{array}$ & 33,5 & 5,8 & NI & 0,03 & NI & 0,01 & 0,14 \\
\hline $\begin{array}{l}\text { Skutskär / Stora Enso } \\
\text { Oyj }\end{array}$ & 36,7 & 0,6 & NI & 0,12 & NI & 0,06 & 0,3 \\
\hline $\begin{array}{l}\text { Kaukas / UPM- } \\
\text { Kymmene Oyj }\end{array}$ & 40,6 & 13,6 & NI & 0,12 & 0,87 & 0,005 & 0,16 \\
\hline $\begin{array}{l}\text { Kymi / uPM- } \\
\text { Kymmene Oyj }\end{array}$ & 48,2 & 13,9 & NI & 0,15 & 0,42 & 0,01 & 0,13 \\
\hline $\begin{array}{l}\text { Pietarsaari / UPM- } \\
\text { Kymmene Oyj }\end{array}$ & 43,2 & 17 & NI & 0,09 & 0,8 & 0,01 & 0,18 \\
\hline $\begin{array}{l}\text { Stora Enso (datos } \\
\text { consolidados) }\end{array}$ & 41,9 & 13,5 & NI & 0,1 & NI & 0,007 & 0,08 \\
\hline $\begin{array}{l}\text { UPM (datos } \\
\text { consolidados) }\end{array}$ & 37 & 12 & NI & 0 & 1 & 0,011 & 0,13 \\
\hline $\begin{array}{l}\text { Media plantas en el } \\
\text { Cono Sur }\end{array}$ & 27,6 & 6,1 & 0,45 & 0,05 & 0,89 & 0,014 & 0,034 \\
\hline $\begin{array}{l}\text { Media plantas en el } \\
\text { exterior }\end{array}$ & 42,9 & 11,71 & & 0,11 & 0,7 & 0,018 & 0,17 \\
\hline BAT - límite inferior & 30 & 8 & 0,3 & $<0,25$ & 0,6 & 0,01 & 0,25 \\
\hline BAT - límite superior & 50 & 23 & 1,5 & & 1,5 & 0,03 & 1 \\
\hline
\end{tabular}

FUENTE INFORMES DE SUSTENTABILIDAD Y PÁGINAS INSTITUCIONALES EN INTERNET

11 La DQO señala la cantidad de oxígeno necesaria para la oxidación de materias orgánicas. Cuanto más alto es su valor, mayor es la concentración de materia orgánica y menor es el contenido de oxígeno en el agua.

12 La рво es utilizada para medir el consumo de oxígeno en el agua, porque indica la cantidad necesaria de ese elemento para oxidar la materia orgánica biodegradable presente en ella. Elevados niveles de Dво interfieren directamente en la calidad del agua, dificultando el proceso de descomposición de algunas substancias y haciendo que ellas permanezcan presentes. 
Otra emisión característica de la producción de celulosa es la liberación de halógeno orgánico adsorbido (AOx). ${ }^{13}$ Positivamente, en todas las plantas seleccionadas las emisiones fueron inferiores al límite determinado por la BAT; y en el caso de las fábricas ubicadas en el Cono Sur, la media de emisión fue inferior a la observada en la media de las plantas en el exterior. El total de sólidos suspensos (TSs) ${ }^{14}$ fue superior solo en la planta de Cenibra. Las otras dos fábricas ubicadas en el Cono Sur, junto con la unidad Kymi, fueron las que presentaron las menores cantidades de emisión. Tanto el fósforo $(\mathrm{P})$ como el nitrógeno $(\mathrm{N})$ son dos componentes presentes en los efluentes de la producción de celulosa. ${ }^{15}$ Los niveles de desecho de fósforo y nitrógeno fijados por la вАт fueron respetados por la mayoría de las unidades seleccionadas.

Evaluando conjuntamente los índices de emisión, se verifica que las plantas extranjeras instaladas en el Cono Sur poseen un desempeño más tolerable que el observado en las filiales del exterior. Entre las tres fábricas analizadas, solamente la de Cenibra presentó índices de emisión preocupantes de descartes de dióxido de azufre en el aire y de sólidos suspendidos en el agua. Las otras dos - Veracel y Fray Bentos- se diferenciaron de las demás con índices más tolerables. Como son unidades recientes, es muy probable que empleen tecnologías más modernas y, por lo tanto, menos dańinas al medioambiente.

La evaluación de conducta ambiental se estableció a partir de la presencia de certificaciones, la divulgación de informes de sustentabilidad u otros documentos semejantes, y considerando si esos informes contenían informaciones de inversiones en mecanismos de control de contaminación (tabla 6).

Como era esperado, la existencia de certificación fue elevada: todas las unidades son certificadas con ISO900 I e ISOI400I, y certificaciones de cadena de custodia Forest Stewardship Council (FSC), y Program for Endorsement of Forestry Certification (PEFC). Además, seis plantas tienen la certificación OHSAS I800I, que identifica aplicación de política de salud y seguridad de los trabajadores. Sobre las inversiones aplicadas en mejoría ambiental, todas las unidades analizadas manifestaron, en sus informes de sustentabilidad, la aplicación de recursos en acciones de carácter ambiental. Sin embargo, solamente la unidad Cenibra y la upM-Kymmene divulgaron el monto invertido. La primera, en 2012, gastó us $\$ 2,24$ millones (o R $\$ 4,57$ millones) en inversiones ambientales, cifra que correspondió al 0,37\% de sus ingresos netos y al 2,21\% de sus inversiones totales. La compañía UPM-Kymmene informó que en 2012 se invirtieron us $\$ 46,16$ millones ( $€ 35$ millones), pero ese valor corresponde al total de inversiones en todas sus unidades de negocios (celulosa, papel, productos forestales y energía). Con relación a la divulgación de los

13 La emisión Aox deriva de la adición de cloro y dióxido de cloro al proceso de blanqueamiento. Varias investigaciones indican que la fuga de AOx puede potencializar el surgimiento de células cancerígenas y de mutaciones, además de otros problemas de toxicidad.

14 El tss mide toda la materia que se conserva en el agua como residuo, incluso después de la evaporación.

15 El fósforo y el nitrógeno son elementos importantes, denominados macronutrientes, que sirven de nutrientes en procesos biológicos. El depósito de altos niveles puede provocar la eutrofización, que es el aumento excesivo de nutrientes en el agua, resultando en el aumento exagerado de algunos organismos (como las algas) y ocasionando problemas de calidad y de abastecimiento. 
niveles de emisión, se señala que las tres plantas extranjeras ubicadas en la región informaron todos los parámetros seleccionados. Sin embargo, algunos datos de las empresas UPM-Kymmene y Stora Enso no fueron publicados en los informes. Es el caso de emisiones de partículas en el aire (Dust kg/Adt) y de totales de sólidos suspendidos en el agua (TSS kg/Adt), que no se publicaron ni en términos consolidados, ni por unidad de producción. La empresa Stora Enso tampoco ha divulgado las emisiones de compuesto reducido de azufre en el aire.

TABLA 6 Indicadores de conducta ambiental, plantas industriales seleccionadas, 2012

\begin{tabular}{|c|c|c|c|c|c|c|c|c|}
\hline \multicolumn{2}{|c|}{ PLANTAS NDUSTRIALES } & \multirow{2}{*}{$\begin{array}{c}\text { ISO } \\
900 I \\
X\end{array}$} & \multirow{2}{*}{$\begin{array}{c}\text { ISO } \\
\text { I 400 I } \\
\mathrm{X}\end{array}$} & \multirow{2}{*}{$\begin{array}{r}\text { FSC } \\
\mathrm{X}\end{array}$} & \multirow{2}{*}{$\begin{array}{c}\text { PEFC } \\
\mathrm{X}\end{array}$} & \multirow{2}{*}{$\begin{array}{l}\text { OHSAS } \\
\text { I } 800 \text { I }\end{array}$} & \multirow{2}{*}{$\begin{array}{l}\text { IS } \\
\mathrm{X}\end{array}$} & \multirow{2}{*}{$\begin{array}{c}\text { INVERSIÓN } \\
\text { EN EL } \\
\text { MEDIO } \\
\text { AMBIENTE } \\
\text { X }\end{array}$} \\
\hline \multirow{3}{*}{$\begin{array}{l}\text { Cono } \\
\text { Sur }\end{array}$} & $\begin{array}{l}\text { Cenibra /JBP Resources } \\
\text { Develop. Co }\end{array}$ & & & & & & & \\
\hline & $\begin{array}{l}\text { Veracel /joint venture } \\
\text { Stora Enso }\end{array}$ & $\mathrm{X}$ & $\mathrm{X}$ & $\mathrm{X}$ & $\mathrm{X}$ & & $\mathrm{X}$ & $\mathrm{X}$ \\
\hline & $\begin{array}{l}\text { Fray Bentos /UPM- } \\
\text { Kymmene }\end{array}$ & $\mathrm{X}$ & $\mathrm{X}$ & $\mathrm{X}$ & $\mathrm{X}$ & $\mathrm{X}$ & & \\
\hline \multirow{8}{*}{ Exterior } & Enocell / Stora Enso & $\mathrm{X}$ & $\mathrm{X}$ & $\mathrm{X}$ & $\mathrm{X}$ & $\mathrm{X}$ & & \\
\hline & Sunilla / Stora Enso & $\mathrm{X}$ & $\mathrm{X}$ & $\mathrm{X}$ & $\mathrm{X}$ & $\mathrm{X}$ & & \\
\hline & Skutskär /Stora Enso & $\mathrm{X}$ & $\mathrm{X}$ & $\mathrm{X}$ & $\mathrm{X}$ & $\mathrm{X}$ & & \\
\hline & $\begin{array}{l}\text { Kaukas / UPM- } \\
\text { Kymmene }\end{array}$ & $\mathrm{X}$ & $\mathrm{X}$ & $\mathrm{X}$ & $\mathrm{X}$ & & & \\
\hline & $\begin{array}{l}\text { Kymi / UPM-Kym- } \\
\text { mene }\end{array}$ & $\mathrm{X}$ & $\mathrm{X}$ & $\mathrm{X}$ & $\mathrm{X}$ & $\mathrm{X}$ & & \\
\hline & $\begin{array}{l}\text { Pietarsaari / UPM- } \\
\text { Kymmene }\end{array}$ & $\mathrm{X}$ & $\mathrm{X}$ & $\mathrm{X}$ & $\mathrm{X}$ & $\mathrm{X}$ & & \\
\hline & $\begin{array}{l}\text { Stora Enso } \\
\text { consolidado }\end{array}$ & $\mathrm{X}$ & $\mathrm{X}$ & $\mathrm{X}$ & $\mathrm{X}$ & $\mathrm{X}$ & $\mathrm{X}$ & $\mathrm{X}$ \\
\hline & $\begin{array}{l}\text { UPM-Kymmene } \\
\text { consolidado }\end{array}$ & $\mathrm{X}$ & $\mathrm{X}$ & $\mathrm{X}$ & $\mathrm{X}$ & $\mathrm{X}$ & $\mathrm{X}$ & $\mathrm{X}$ \\
\hline
\end{tabular}

FUENTE INFORMES DE SUSTENTABILIDAD (2OI 2)

Perfil ambiental de las compañías de celulosa del Cono Sur

La finalidad de esta sección es establecer el perfil ambiental de las empresas localizadas en el Cono Sur. En la figura 1 se muestran los principales centros productivos de celulosa de mercado en el Cono Sur, según la posición en 2013. En la región, en ese ańo, el grupo de las empresas de celulosa de mercado estaba formado por nueve compañías que, con sus respectivas plantas industriales, tenían una capacidad instalada de 18,8 millones de toneladas/año (figura 2 y tabla 7). En 2013, esas unidades fueron responsables por $84 \%$ de la producción de celulosa de la región. En total 
fueron seleccionadas 17 unidades industriales en Brasil, Chile, Argentina y Uruguay. ${ }^{16}$ Además, como en el análisis anterior, las informaciones se refieren al ańo $2012 .{ }^{17}$

FIGURA 2 Localización y capacidad instalada de las principales unidades de producción de celulosa, Cono Sur, 2013

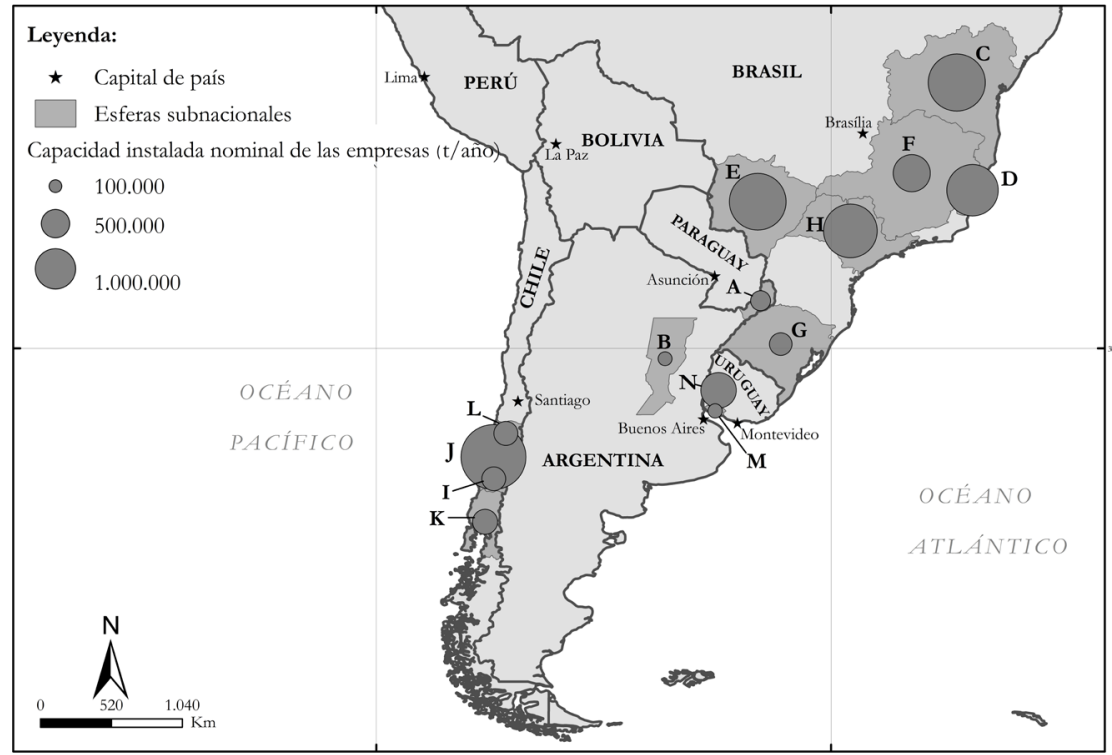

FUENTE CARTOGRAFÍA ESRL

TABla 7 Características de las principales compañías de celulosa del Cono Sur, 2013

\begin{tabular}{|c|c|c|c|c|c|}
\hline $\begin{array}{l}\text { ABREVIA- } \\
\text { TURAS }\end{array}$ & COMPAÑÍA & UNIDAD & PAÍs & $\begin{array}{c}\text { ESFERA } \\
\text { SUBNACIONAL }\end{array}$ & $\begin{array}{c}\text { CAPACIDAD } \\
\text { INSTALADA } \\
\text { (I OOOT })\end{array}$ \\
\hline A & Arauco s/A & Alto Paraná & \multirow{2}{*}{ Argentina } & Missiones & 350 \\
\hline B & Celulosa Argentina s/A & C. Bermudez & & Santa Fé & 210 \\
\hline \multirow{2}{*}{$\mathrm{C}$} & Veracel Celulose s/A & Veracel & \multirow{6}{*}{ Brasil } & \multirow{2}{*}{ Bahia } & 1.100 \\
\hline & Suzano Papel e Celulose s/A & Mucuri & & & 1.740 \\
\hline $\mathrm{D}$ & Fibria Celulose s/A & Aracruz & & Espírito Santo & 2.300 \\
\hline \multirow{2}{*}{ E } & Fibria Celulose s/A & Três Lagoas & & \multirow{2}{*}{ Mato Grosso do Sul } & 1.300 \\
\hline & Eldorado Brasil Celulose s/A & Eldorado & & & 1.500 \\
\hline $\mathrm{F}$ & $\begin{array}{l}\text { JBP Resource and } \\
\text { Development Co. }\end{array}$ & Cenibra & & Minas Gerais & 1.190 \\
\hline
\end{tabular}

(continúa)

16 En la selección inicial había otras tres empresas: Celulosa Argentina y Fanapel (Argentina) y Lwarcel Celulose (Brasil), pero fueron excluidas, pues no proporcionaron un número mínimo de informaciones de emisión y tampoco publicaron informes de sustentabilidad.

17 Sin embargo, en algunos casos los datos se refieren a las emisiones en el año 2009. Además, en particular, en los dos cálculos de las emisiones medias se computaron los datos de la fábrica Veracel, cuya composición accionaria es de un 50\% de capital nacional y un 50\% de capital extranjero. 
(continuación)

\begin{tabular}{|c|c|c|c|c|c|}
\hline $\begin{array}{l}\text { ABREVIA- } \\
\text { TURAS }\end{array}$ & COMPAÑÍA & UNIDAD & PAís & $\begin{array}{c}\text { ESFERA } \\
\text { SUBNACIONAL }\end{array}$ & $\begin{array}{l}\text { CAPACIDAD } \\
\text { INSTALADA } \\
\text { (I OOOT) }\end{array}$ \\
\hline \multirow{2}{*}{ G } & \multirow{2}{*}{ CMPC S/A } & Celulose & \multirow{6}{*}{ Brasil } & \multirow{2}{*}{ Rio Grande do Sul } & \multirow{2}{*}{450} \\
\hline & & Riograndense & & & \\
\hline \multirow{4}{*}{$\mathrm{H}$} & Fibria Celulose s/A & Jacareí & & \multirow{4}{*}{ São Paulo } & 1.100 \\
\hline & Suzano Papel e Celulose s/A & Suzano & & & 500 \\
\hline & Suzano Papel e Celulose S/A & Limeira & & & 650 \\
\hline & $\begin{array}{l}\text { Lwarcel Celulose e Papel } \\
\text { Ltda. }\end{array}$ & Lwarcel & & & 250 \\
\hline I & CMPC S/A & Pacífico & \multirow{7}{*}{ Chile } & Araucanía & 500 \\
\hline \multirow{4}{*}{$\mathrm{J}$} & Arauco S/A & Arauco & & \multirow{4}{*}{ Bío Bío } & 790 \\
\hline & Arauco S/A & Nueva Aldeia & & & 1.027 \\
\hline & CMPC S/A & Laja & & & 360 \\
\hline & CMPC S/A & Santa Fé & & & 1.490 \\
\hline K & Arauco S/A & Valdivia & & Los Ríos & 550 \\
\hline $\mathrm{L}$ & Arauco S/A & Licancel & & Maule & 150 \\
\hline M & Fanapel s/A & Fanapel & \multirow{2}{*}{ Uruguay } & Colonia & 180 \\
\hline $\mathrm{N}$ & UPM - Kymmene Oyj & Fray Bentos & & Río Negro & 1.100 \\
\hline
\end{tabular}

FUENTE SPEROTTO (2OI4)

El primer conjunto de índices remite a los contaminantes del aire (tabla 8). Como ya se expuso en la sección anterior, Cenibra fue la que más emitió partículas sólidas al aire (Dust). Otra unidad con elevados niveles fue la de Jacareí, de la compañía Fibria. Entre las compañías, llaman la atención los niveles de Arauco y de смPC, también más elevado que el límite superior. Sin embargo, en el caso de CMPC, se debe considerar que la emisión también agrega la proveniente de la producción de papel. Lo mismo ocurrió con la planta de Mucuri, de Suzano Papel e Celulose, que, aunque concentre su producción en la celulosa, es una unidad industrial que fabrica papeles y presentó un nivel de contaminación mayor que el establecido. Es interesante apuntar que, en promedio, la emisión de partículas de las plantas extranjeras fue inferior a la de las unidades y compañías de la región, revelando, en el caso de las primeras, un desempeño más tolerable. Las emisiones de dióxido de azufre $\left(\mathrm{SO}_{2}\right)$ fueron superiores al límite definido por la BAT, tanto en la media de las plantas del Cono Sur como en las extranjeras. Las emisiones consolidadas de ambas empresas chilenas también sobrepasaron ese límite. Los menores niveles de contaminación se verificaron en las unidades Veracel, Três Lagoas, Fray Bentos y Aracruz. En cuanto a las emisiones de compuesto de azufre reducido (TRS), se constata que en su mayoría las plantas respetaron los límites BAT; incluso, muchas de ellas presentaron emisiones por debajo del límite inferior. Una de las hipótesis es que la fácil percepción y el amplio alcance de esa emisión hacen que las empresas busquen medios de atenuar los niveles de ese componente en el aire. 
TABLA 8 | Índices de emisión en el aire, unidades de producción seleccionadas, Cono Sur, 2012

\begin{tabular}{|c|c|c|c|c|}
\hline $\begin{array}{c}\text { PLANTAS INDUSTRIALES/ } \\
\text { COMPAŃÍAS }\end{array}$ & $\begin{array}{c}\text { DUST } \\
(\text { KG/ADT })\end{array}$ & $\begin{array}{l}\text { SO2 (KG/ } \\
\text { ADT) }\end{array}$ & $\begin{array}{l}\text { NOX }(\text { KG/ } \\
\text { ADT })\end{array}$ & $\begin{array}{c}\text { TRS (KG/ } \\
\text { ADT) }\end{array}$ \\
\hline Cenibra / JBP Resources Develp. Co & 1,31 & 2,61 & 0,13 & 0,02 \\
\hline $\begin{array}{l}\text { Veracel / Fibria Celulose s/A e Stora } \\
\text { Enso Oyj. }\end{array}$ & 0,23 & 0,01 & 0,81 & 0,01 \\
\hline Fray Bentos / UPM-Kymmene Oyj. & 0,13 & 0,05 & 1,6 & 0 \\
\hline Aracruz / Fibria Celulose s/A & 0,19 & 0,06 & 0,2 & 0,01 \\
\hline Jacareí / Fibria Celulose s/A & 1,25 & 0,1 & 0,67 & 0,02 \\
\hline Três Lagoas/ Fibria Celulose s/A & 0,22 & 0,04 & 1,52 & 0,06 \\
\hline Mucuri / Suzano Papel e Celulose s/A & 0,79 & 0,34 & 1,35 & 0,09 \\
\hline Fibria Celulose s/A consolidado & 0,49 & 0,1 & 0,68 & 0,03 \\
\hline Arauco S/A consolidado & 0,76 & 1 & 1,9 & 0,1 \\
\hline CMPC s/A consolidado & 0,99 & 1,48 & 1,7 & \\
\hline Media compañías del Cono Sur & 0,61 & 0,39 & 1,1 & 0,05 \\
\hline $\begin{array}{l}\text { Media de las plantas extranjeras en el } \\
\text { Cono Sur }\end{array}$ & 0,56 & 0,89 & 0,85 & 0,01 \\
\hline Media compañías brasileńas & 0,5 & 0,15 & 0,94 & 0,04 \\
\hline Media compañías chilenas & 0,87 & 1,24 & 1,8 & 0,1 \\
\hline BAT - límite inferior & 0,2 & 0,2 & 1 & 0,1 \\
\hline BAT - límite superior & 0,5 & 0,4 & 1,5 & 0,2 \\
\hline
\end{tabular}

FUENTE INFORMES DE SUSTENTABILIDAD Y PÁGINAS INSTITUCIONALES EN INTERNET

Con respecto a los flujos de efluentes en el agua (tabla 9), el límite superior de la BAT no fue sobrepasado por ninguna de las plantas o las compañías. Entre las tres unidades extranjeras, la que presentó la mayor emisión fue Cenibra. Comparando las emisiones medias de los dos grupos, las empresas nacionales depositaron, otra vez, un volumen superior al de las extranjeras. Los parámetros de demanda química (DQO) y bioquímica de oxígeno (DBO) indican los niveles de oxigenación y la calidad del agua. En el caso de la DQO, todas las unidades presentaron un desempeño tolerable, pero superior al límite inferior BAT. Las mejores performances se observaron en las plantas de Fray Bentos y Veracel. En contrapunto, el desempeño más preocupante se verificó en la unidad Aracruz. En lo que se refiere a la DBО, los niveles de emisión permanecieron por debajo del límite superior. Sin embargo, en la unidad Aracruz y, por consiguiente, en la empresa Fibria, los niveles fueron muy elevados, provocando posibles problemas en materia de calidad. Acerca de los depósitos de halógenos orgánicos adsorbidos (AOx), todas las unidades y compañías lanzaron cantidades menores que las determinadas por la BAT. El límite superior de descartes totales de sólidos suspendidos (TSS) fue sobrepasado, principalmente, por las compañías nacionales. Esa contaminación se vuelve más preocupante porque las unidades industriales son de gran escala, por lo que el valor absoluto de esos descartes es muy elevado. Los lanzamientos de fósforo excedieron los límites BAT en tres plantas industriales: Três Lagoas, Aracruz y Celulose Riograndense. Tal resultado 
también se constató en las demás empresas de la región. La performance en los descartes de nitrógeno fue más adecuada a los límites BAT, con volúmenes incluso menores que el corte inferior.

En síntesis, el desempeño ambiental de las plantas extranjeras ubicadas en el Cono Sur es más tolerable que el observado en las plantas y compañías nacionales.

TABLA 9 Índices de emisión en el agua, unidades de producción seleccionadas, Cono Sur, 2012

\begin{tabular}{|c|c|c|c|c|c|c|c|}
\hline $\begin{array}{l}\text { PLANTAS INDUSTRIALES/ } \\
\text { COMPAŃÍAS }\end{array}$ & $\begin{array}{c}\text { FLUJO } \\
\text { EFLUENTE } \\
(\text { (M3/ADT) }\end{array}$ & $\begin{array}{l}\text { DQO } \\
\text { (KG/ } \\
\text { ADT) }\end{array}$ & $\begin{array}{l}\text { DBO } \\
(\mathrm{KG} / \\
\text { ADT })\end{array}$ & $\begin{array}{l}\text { AOX } \\
\text { (KG/ } \\
\text { ADT) }\end{array}$ & $\begin{array}{c}\text { TSS } \\
(\mathrm{KG} / \\
\text { ADT })\end{array}$ & $\begin{array}{c}\mathbf{P}(\mathrm{KG} / \\
\mathrm{ADT})\end{array}$ & $\begin{array}{l}\mathbf{N}(\mathrm{KG} / \\
\mathbf{A D T})\end{array}$ \\
\hline Cenibra / JвP Resources Develp. Co & 42,4 & 8,8 & 0,99 & 0,07 & 1,9 & 0 & 0 \\
\hline $\begin{array}{l}\text { Veracel / joint venture Stora Enso y } \\
\text { Fibria }\end{array}$ & 21,2 & 5,5 & 0,23 & 0,05 & 0,56 & 0,02 & 0,05 \\
\hline Fray Bentos / UPM-Kymmene Oyj. & 19,3 & 4 & 0,14 & 0,03 & 0,2 & 0,02 & 0,05 \\
\hline Celulose Riograndense / CMPC s/A & 29,7 & NI & 0,11 & 0,09 & NI & 0,08 & 0,08 \\
\hline Aracruz / Fibria Celulose s/A & 34,1 & 14,6 & 2,84 & 0,09 & 2,06 & 0,07 & 0,3 \\
\hline Jacareí / Fibria Celulose s/A & 22,5 & 8,1 & 0,57 & 0,05 & 2,63 & 0,01 & 0 \\
\hline Três Lagoas / Fibria Celulose s/A & 37,2 & 6,9 & 0,73 & 0,08 & 3,4 & 0,07 & 0,09 \\
\hline Mucuri / Suzano Papel e Celulose s/A & 25,7 & 12,8 & 1,33 & 0,08 & NI & NI & NI \\
\hline Fibria Celulose s/A consolidado & 32,8 & 11,2 & 1,78 & 0,08 & 2,59 & 0,06 & 0,18 \\
\hline Arauco S/A consolidado* & 48 & 12,1 & 0,76 & 0,1 & 1,62 & 0,04 & 0,09 \\
\hline CMPC s/A consolidado & NI & 10,3 & 1,57 & 0,07 & 1,08 & 0,05 & 0,15 \\
\hline Media compañías del Cono Sur & 32,8 & 10,9 & 1,21 & 0,08 & 2,23 & 0,05 & 0,13 \\
\hline $\begin{array}{l}\text { Media plantas extranjeras en el } \\
\text { Cono Sur }\end{array}$ & 27,6 & 6,1 & 0,45 & 0,05 & 0,89 & 0,01 & 0,03 \\
\hline Media compañías brasileñas & 25,94 & 9,34 & 1,08 & 0,06 & 1,4 & 0,04 & 0,11 \\
\hline Media compañías chilenas & 48 & 11,19 & 1,17 & 0,09 & 1,35 & 0,04 & 0,12 \\
\hline BAT - límite inferior & 30 & 8 & 0,3 & $<0,25$ & 0,6 & 0,01 & 0,25 \\
\hline BAT - límite superior & 50 & 23 & 1,5 & & 1,5 & 0,03 & 1 \\
\hline
\end{tabular}

NOTA * DATOS DE EMISIÓN DE 2009.

FUENTE INFORMES DE SUSTENTABILIDAD Y PÁGINAS INSTITUCIONALES EN INTERNET

La conducta ambiental, como se ha destacado, se refiere a la definición y la aplicación de mecanismos de comportamiento ambiental, los cuales buscan comunicar, interna y externamente, las prácticas de gestión ambiental de la empresa. Prácticamente todas las unidades industriales poseen las certificaciones seleccionadas: el 100\% son certificadas por las Iso9oo I e ISO I 400 I; el 100\% tienen certificación FSC de cadena de custodia; y el 88\% (15 unidades) poseen certificación PEFC de cadena de custodia. De esas últimas, seis fueron obtenidas a través del Cerflor certificación brasileña de productos forestales-, y ocho por el Certfor -certificación chilena de productos forestales-, ambas habilitadas por el PEFC (tabla 10). Además, 
seis unidades poseen la certificación OHSAS I 800 I, sello que indica la adopción de políticas internas de salud y seguridad de los empleados.

TABla io | Indicadores de conducta ambiental, plantas industriales, Cono Sur, 2012

\begin{tabular}{|c|c|c|c|c|c|c|c|c|}
\hline PLANT & $\begin{array}{l}\text { AS INDUSTRIALES/ } \\
\text { COMPAŃÍAS }\end{array}$ & $\begin{array}{c}\text { ISO } \\
900 \text { I }\end{array}$ & $\begin{array}{l}\text { ISO } \\
\text { I } 400 \text { I }\end{array}$ & FSC & PEFC & $\begin{array}{l}\text { OHSAS } \\
\text { I } 800 \text { I }\end{array}$ & IS & $\begin{array}{l}\text { INVERSIÓN } \\
\text { EN EL } \\
\text { MEDIO }\end{array}$ \\
\hline & Cenibra / JBP R\&D & $\mathrm{X}$ & $\mathrm{X}$ & $\mathrm{X}$ & $\mathrm{X}$ & & $\mathrm{X}$ & $\mathrm{X}$ \\
\hline Extran- & $\begin{array}{l}\text { Fray Bentos / UPM- } \\
\text { Kymmene }\end{array}$ & $\mathrm{X}$ & $\mathrm{X}$ & $\mathrm{X}$ & $\mathrm{X}$ & $\mathrm{X}$ & $\mathrm{X}$ & $\mathrm{X}$ \\
\hline & $\begin{array}{l}\text { Veracel / joint verture } \\
\text { Fibria Celulose e Stora } \\
\text { Enso }\end{array}$ & $\mathrm{X}$ & $\mathrm{X}$ & $\mathrm{X}$ & $\mathrm{X}$ & & $\mathrm{X}$ & $\mathrm{X}$ \\
\hline & $\begin{array}{l}\text { Aracruz / Fibria } \\
\text { Celulose }\end{array}$ & $\mathrm{X}$ & $\mathrm{X}$ & $\mathrm{X}$ & $\mathrm{X}$ & & & \\
\hline & \begin{tabular}{|l|} 
Jacareí / Fibria \\
Celulose
\end{tabular} & $\mathrm{X}$ & $\mathrm{X}$ & $\mathrm{X}$ & & & & \\
\hline & $\begin{array}{l}\text { Três Lagoas / Fibria } \\
\text { Celulose }\end{array}$ & $\mathrm{X}$ & $\mathrm{X}$ & $\mathrm{X}$ & $\mathrm{X}$ & & & \\
\hline & $\begin{array}{l}\text { Mucuri / Suzano Papel } \\
\text { e Celulose }\end{array}$ & $\mathrm{X}$ & $\mathrm{X}$ & $\mathrm{X}$ & $\mathrm{X}$ & $\mathrm{X}$ & & \\
\hline & $\begin{array}{l}\text { Celulose Riograndense } \\
\text { / CMPC }\end{array}$ & $\mathrm{X}$ & $\mathrm{X}$ & $\mathrm{X}$ & $\mathrm{X}$ & & $\mathrm{X}$ & $\mathrm{X}$ \\
\hline & Laja / CMPC & $\mathrm{X}$ & $\mathrm{X}$ & $\mathrm{X}$ & $\mathrm{X}$ & $\mathrm{X}$ & & \\
\hline & Pacífico / CMPC & $\mathrm{X}$ & $\mathrm{X}$ & $\mathrm{X}$ & $\mathrm{X}$ & $\mathrm{X}$ & & \\
\hline Nacionales & Santa Fé / CMPC & $\mathrm{X}$ & $\mathrm{X}$ & $\mathrm{X}$ & $\mathrm{X}$ & $\mathrm{X}$ & & \\
\hline $\begin{array}{l}\text { der Cono } \\
\text { Sur }\end{array}$ & Alto Paraná / Arauco & $\mathrm{X}$ & $\mathrm{X}$ & $\mathrm{X}$ & & $\mathrm{X}$ & & \\
\hline & Arauco / Arauco & $\mathrm{X}$ & $\mathrm{X}$ & $\mathrm{X}$ & $\mathrm{X}$ & & & \\
\hline & Constitución / Arauco & $\mathrm{X}$ & $\mathrm{X}$ & $\mathrm{X}$ & $\mathrm{X}$ & & & \\
\hline & Licancel / Arauco & $\mathrm{X}$ & $\mathrm{X}$ & $\mathrm{X}$ & $\mathrm{X}$ & & & \\
\hline & Nueva Aldea / Arauco & $\mathrm{X}$ & $\mathrm{X}$ & $\mathrm{X}$ & $\mathrm{X}$ & & & \\
\hline & Valdivia / Arauco & $\mathrm{X}$ & $\mathrm{X}$ & $\mathrm{X}$ & $\mathrm{X}$ & & & \\
\hline & Fibria consolidado & & & & & & $\mathrm{X}$ & $\mathrm{X}$ \\
\hline & $\begin{array}{l}\text { Suzano P. e Celulose } \\
\text { consolidado }\end{array}$ & & & & & & $\mathrm{X}$ & $\mathrm{X}$ \\
\hline & Arauco consolidado & & & & & & $\mathrm{X}$ & $\mathrm{X}$ \\
\hline & CMPC consolidado & & & & & & $\mathrm{X}$ & $\mathrm{X}$ \\
\hline
\end{tabular}

FUENTE INFORMES DE SUSTENTABILIDAD (2OI2)

Con respecto a las inversiones en controles ambientales, todas las compañías del Cono Sur, así como las empresas extranjeras en la región, afirmaron haber invertido en mejorías ambientales en el año 2012. Tales inversiones, cuando divulgadas, consideraron recursos para disminuir el consumo de agua, eliminar olores y ejecutar permanentemente el monitoreo de la fauna, de la flora y de los recursos hídricos. Entre las plantas y compañías, las que informaron los valores aplicados fueron: 
Cenibra (us $\$ 2,24$ millones, 2,2\% de las inversiones totales); Celulose Riograndense (us $\$ 1,15$ millones), Suzano Papel e Celulose (us $\$ 14,6$ millones), Fibria (us $\$ 104,1$ millones, en proyectos y tratamiento ambiental) y Arauco (US\$19 millones, 1,8\% de las inversiones totales). ${ }^{18}$

En cuanto a la divulgación de los niveles de emisión, como ya se señaló, las tres plantas extranjeras ubicadas en el Cono Sur publicaron, en sus respectivos informes o reportes de sustentabilidad, todos los parámetros elegidos en el análisis de desempeño ambiental. En el conjunto de las empresas nacionales, aunque la mayoría haya informado los datos, en tres hubo deficiencias: (i) las informaciones de 2012 de la compañía Fibria fueron obtenidas en el is de 2013, pues en el is de 2012 algunos datos no fueron divulgados; (ii) del mismo modo, la empresa Arauco no informó algunos índices de emisión, los cuales fueron capturados con base en el año de 2009; y (iii) CMPC solamente informa los datos consolidados de todas sus plantas en distintas áreas de negocios. En líneas generales, exceptuando los casos recién citados, no se constataron diferencias significativas entre las empresas nacionales y las plantas extranjeras. Una probable razón es la creciente presión, principalmente internacional, por más certificaciones y sellos que informen la procedencia de las materias primas y la especificación de los procesos de producción empleados.

\section{Consideraciones finales}

En los últimos veinte años hubo importantes cambios en el segmento de celulosa de mercado. Además de las alteraciones tecnológicas a lo largo de su cadena de producción, se constata nítidamente el desplazamiento del centro productivo desde los países desarrollados a los países en desarrollo. Entre los últimos destacan Brasil, Chile y, más recientemente, Uruguay. Actualmente, la región es responsable del 30\% de la producción mundial y es la primera exportadora mundial de pasta. Además, hasta 2020 se esperan importantes inversiones, tanto en nuevas plantas como en proyectos de ampliación de unidades industriales ya existentes. En los dos casos, hay una presión muy fuerte para expandir la escala de producción, una vez que la capacidad nominal instalada de esas nuevas plantas industriales supere el millón de toneladas/año.

Aunque una parte de esa expansión resulte del proprio proceso de desarrollo de los países señalados — asociado a la difusión de procesos y tecnologías vinculados a las metas de autoabastecimiento y de crecimiento industrial—, la presión respecto de los impactos ambientales de esa actividad, de elevado potencial contaminante, es un incentivo adicional a su deslocalización desde economías desarrolladas a las economías emergentes o en desarrollo.

A partir de esa problemática, el objetivo del artículo fue investigar la posible existencia de diferencias de comportamiento ambiental, las cuales pudiesen indicar la formación de un paraíso de contaminación. Como se señaló, la creación de esos 
paraísos suele ser entendida a partir del encadenamiento de elementos relacionados con las estrategias de localización y la presión ejercida por una política ambiental más rigorosa, de donde resulta una nueva disposición productiva. Sin embargo, algunos de los aspectos presentes en esa dinámica son complejos -como la comparación del nivel de rigidez de las reglas y leyes ambientales entre los países- y otros son opacos, como los problemas de retórica corporativa.

Con la intención de proponer un instrumento más factible de comparación, se eligió comparar los indicadores de desempeńo y de conducta ambiental a partir del modelo de evaluación sector-control-conducta-desempeño. El objetivo de ese modelo fue evitar algunos de los obstáculos indicados para la comprobación de la hipótesis de paraísos de contaminación. Para alcanzar ese propósito se aplicaron tres indicadores ambientales: uno de control, BAT, utilizado para fijar límites de emisión en las reglas y las normas ambientales, y otros dos -conducta y desempeño- centrados en las prácticas de las empresas. La aplicación de esos indicadores consideró dos conjuntos de unidades de producción: uno formado por las plantas extranjeras ubicadas en el Cono Sur, con sus filiales en el exterior, y otro con esas primeras y las demás empresas de la región.

En forma sintética, se observaron diferencias leves de conducta ambiental entre las plantas extranjeras y las nacionales; sin embargo, hubo diferencias importantes en el desempeńo. Las fábricas extranjeras ubicadas en el Cono Sur presentaron, en general, una performance ambiental superior a la de sus propias filiales en el exterior y a la de las plantas nacionales de la región. Los parámetros más tolerables de las plantas extranjeras, muy probablemente, están relacionados con su tiempo de existencia (son fábricas nuevas) y, por consiguiente, con el empleo de tecnologías más modernas y más amigables con el medioambiente, como aquellas disponibles en sus países de origen. Por lo tanto, la HPC en la producción de celulosa en el Cono Sur no se confirmó.

No obstante, algunos aspectos deben ser destacados. Primero, en el conjunto de empresas del Cono Sur, el desempeño de las chilenas es inferior al de las brasileñas y uruguaya. Al considerarse que son justamente las chilenas las que están expandiéndose en la región, preocupa el desempeño general de las plantas industriales. En 2009, la compañía СмCP se volvió propietaria de la unidad de la Celulose Riograndense, en el sur de Brasil, y en 2014 empezó a operar la segunda gran planta uruguaya, la Montes del Plata, una joint venture de la chilena Arauco y la suecofinlandesa Stora Enso. Segundo, los canales de comunicación de las compañías nacionales necesitan ser mejorados, en razón de las fallas o ausencias de algunos indicadores. Y, por fin, la elevada capacidad instalada de las nuevas fábricas, que superan el millón de toneladas/año.

$\mathrm{Si}$, por un lado, se confirma que tanto el proceso como la maquinaria transferidos por las empresas extranjeras a la región corresponden a la tecnología más actual empleada en la producción de pasta, por otro lado existe la necesidad de considerarse el tamaño de esas plantas y, por consecuencia, la emisión absoluta de contaminantes lanzados al aire y al agua, además de otros impactos paralelos, como los cambios en el paisaje y los efectos causados por el monocultivo forestal. Por ejemplo, el requerimiento de grandes macizos forestales, aunque estén formados 
por bosques totalmente certificados, desequilibra fuertemente el medioambiente y altera la calidad y la cantidad de los recursos hídricos, la fauna, la flora y el paisaje, como también ocurre con otros monocultivos.

El hecho de no comprobarse la HPC en la producción de celulosa del Cono Sur solo indica que algunas unidades de producción, en este caso las extranjeras, presentan un comportamiento ambiental más tolerable. De esa forma, aunque la HPC no haya sido comprobada, no se puede negar que en la región hay un inminente efecto contaminante en formación, básicamente como consecuencia de la presencia de tantas instalaciones industriales de gran escala, ratificando la idea de efecto contaminante en formación.

Finalmente, a pesar de no confirmarse la HPC, se considera que el modelo propuesto -sector-control-conducta-desempeño- es adecuado para analizar el efecto de la aplicación de reglas ambientales -rígidas o más blandas- sobre el comportamiento de las empresas. Su aplicación permite, a través de datos de emisión e informaciones de gestión, monitorear los niveles de emisión y los esfuerzos de ajuste a los criterios de certificación, en especial en las empresas que actúan en segmentos de alto potencial contaminante y que están fuertemente volcadas al mercado externo.

\section{Agradecimientos}

La autora agradece a los dos revisores anónimos por los comentarios y sugerencias que contribuyeron a perfeccionar este artículo.

\section{Referencias bibliográficas}

Abreu, M. C. S. de (2001). Modelo de avalição da estratégia ambiental: uma ferramenta para a tomada de decisão. Tese, Universidade Federal de Santa Catarina (UFSC), Florianópolis. http://www.lgti.ufsc.br/public/monica.pdf

Arauco (2009). Reporte de sustentabilidad. http://www.arauco.cl

Arauco (2012). Reporte de sustentabilidad. http://www.arauco.cl

Associação Brasileira de Celulose e Papel (Bracelpa) (2013). Datos del Sector. http://www. bracelpa.org.br.

Biazus, A., Da Hora, A. B. \& Leite, B. G. P. (2010). Panorama de mercado: celulose. BNDES Setorial 32, 311-370. http://www.BNDES.gov.br/

Birdsall, N. \& Wheeler, D. (1993) Trade policy and industrial pollution in Latin America: where are the pollution havens? Journal of Environment and Development, 2(1), 137149. http://jed.sagepub.com/content/2/1/137.abstract

Brunnermeier, S. B. \& Levinson, A. (2004). Examining the evidence on environmental regulations and industry location. The Journal of Environment Development, 13(1), 6-41. http://jed.sagepub.com/content/13/1/6

Celulosa Argentina (2013) [sitio institucional web]. http://www.celulosaargentina.com.ar

Celulose Riograndense (2012). Relatório de sustentabilidade. http://www.celuloseriograndense. com.br 
Cenibra (2012). Relatório de sustentabilidade (2012). http://www.cenibra.com.br

Chapman, K. (1982) Environmental policy and industrial location in the United States. En R. Flowerdew (ed.), Institutions and Geographical Patterns (pp. 141-168). Londres: Croom Helm.

CMPC Empresas (2012). Reporte de desarrollo sostenible. http://www.cmpc.cl

Dean, J. (1992). Trade and the environment: a survey of the literature. World Development Report, wPS n. 966. http://documents.worldbank.org/curated/en/1992/08/699608/ trade-environment-survey-literature

Dean, J. M., Lovely, M. E. \& Wang, H. (2009). Are foreign investors attracted to weak environmental regulations? Evaluating the evidence from China. Journal of Development Economics, 90(1), 1-13. http://dx.doi.org/10.1016/j.jdeveco.2008.11.007

Eldorado Brasil (2013). [sitio institucional web]. http://www.eldoradobrasil.com.br.

European Commission (2013). Best Available Techniques (ВАT). Reference document for the production of pulp, paper and board. Institute for Prospective Technological Studies, Sustainable Production and Consumption Unit, and European IPPC Bureau. Final Draft. http://eippcb.jrc.ec.europa.eu/reference/BREF/PP_BREF_FD_07_2013.pdf

Fanapel (2013). [sitio institucional web]. http://www.fanapel.com.uy

Fibria Celulose (2013). Relatório de sustentabilidade. http://www.fibria.com.br

Gallagher, K. S. (2008). Foreign direct investment and clean technology leapfrogging in China. En K. P. Gallagher, Handbook on trade and the Environment (pp. 147-155). Cheltenham: Edward Elgar.

Gray, K. R. (2002). Foreign direct investment and environmental impacts Is the debate over? Review of European Community \& International Environmental Law, 11(3), 306-313. http://onlinelibrary.wiley.com/doi/10.1111/1467-9388.00329/abstract

Hayter, R. (1997). The dynamics of industrial location: the factory, the firm and the production system. Londres: Ed. Wiley.

Indústria Brasileira de Árvores (IBÁ) (2015). Relatório. http://iba.org/pt/biblioteca-iba/ publicacoes

Jänicke, M., Binder, M. \& Mönch, H. (1997). "Dirty Industries”: Patterns of change in industrial countries. Environmental and Resource Economics, 9(4), 467-491. http:// link.springer.com/article/10.1023/A\%3A1026497608363

Leonard, J. J. (1988). Pollution and struggle for the world product: multinational corporations, environment and international comparative advantage. Cambridge, uK: Cambridge University Press.

Levinson, A. (1996). Environmental regulation and industrial location: International and domestic evidence. En J. N. Bragwati \& R. E. Hudec, Fair trade and harmonization: prerequisites for free trade? (vol. 1, pp. 429-457). Cambridge, мA: The мIт Press.

Low, P. \& Yeats, A. (1992). Do "Dirty" industries migrate? En P. Low (ed.), World Bank Discussion Paper, (159), 89-103. http://go.worldbank.org/X5YU9AKL90

Lwarcel Celulose (2013). [sitio institucional web]. http://www.lwarcel.com.br

Lyne, J. (1990). Service taxes, international site selection and the green movement dominate executives political focus. Site Selection. http://www.developmentalliance.com/docu/ pdf/40480.pdf 
Meyer, S. M. (1995). The economic impact of environmental regulation. Journal of Environmental Law and Practice, 3(2), 4-15. http://crywolfproject.org/evidence/ economic-impact-environmental-regulation

Mulatu, A., Gerlagh, R., Rigby, D. \& Wossink, A. (2010). Environmental regulation and industry location in Europe. Environmental and Resource Economics, 45(4), 459-479. http://link.springer.com/article/10.1007/s10640-010-9357-6

Organización de las Naciones Unidas para la Alimentación y la Agricultura (FAO) (2016). Datos FAOSTAT. http:// http://faostat3.fao.org

Porter, G. (1999). Trade competition and pollution standards: "Race to the Bottom" or "Stuck at the Bottom". Journal of Environment and Development, 8(2), 133-151. http://jed. sagepub.com/content/8/2/133

Smarzynska, B. K \& Wei, S. (2001). Pollution havens and foreign direct investment: dirty secret or popular myth? National Bureau of Economic Research, Working Paper n. 8465. http://www.efi.int/portal/virtual_library/publications/proceedings/36/

Sperotto, F. Q. (2012). Um panorama da matriz produtiva de celulose no Cone Sul: caracterização, configuração e produção. Indicadores Econômicos, 39(4), 129-144. http://revistas.fee.tche.br/index.php/indicadores/article/view/3073

Sperotto, F. Q. (2014a). As regulamentaçōes ambientais redefinindo a geografia da produção: o estudo de caso da produção de celulose no Cone Sul. Tese, Universidade Federal do Rio Grande do Sul (UfRGS). http://hdl.handle.net/10183/95376

Sperotto, F. Q. (2014b). A expansão do setor de celulose de mercado no Brasil: condicionantes e perspectivas. Indicadores Econômicos, 41(4), 85-100. http://revistas.fee.tche.br/index. php/indicadores/article/view/3073

Sperotto, F. Q. (2016). Celulose de mercado. FEE Setorial (Porto Alegre), (1). http://www.fee. rs.gov.br/wp-content/uploads/2016/11/20161116fee-setorial-celulose-de-mercadocompressed.pdf

Stafford, H. A. (1977). Environmental regulations and the location of u.s. manufacturing: speculations. Geoforum, 8(5-6), 243-248. http://dx.doi.org/10.1016/00167185(77)90024-0

Stafford, H.A. (1985). Environmental protection and industrial location. Annals of the Association of American Geographers, 75(2), 227-240. http://www.jstor.org/stable/2562564

Stora ENso. (2012). Annual Report. http://www.storaenso.com

Suzano Papel e Celulose (2012). Relatório de sustentabilidade. http://www.suzano.com.br

Taylor, M. S. (2005). Unbundling the pollution haven hypothesis. Discussion Paper n. 15, University of Calgary, Canada. http://www.econ.ucalgary.ca/research/research.htm.

Upm. (2012). Annual Report. http://www.upm.com

Veracel Celulose (2012). Relatório de sustentabilidade. http://www.veracel.com.br

Zarsky, L. (1999). Havens, halos and spaghetti: untangling the evidence about foreign direct investment and the environment. Conference on Foreign Direct Investment and the Environment, OCDE. http://www.oecd.org/daf/internationalinvestment/investmentfordevelopment/ foreigndirectinvestmentandtheenvironment.htm. 